\title{
Article \\ Design Of Acoustical Bessel-Like Beam Formation By Tunable Angular Spectrum In Soret Zone Plate Lens
}

\author{
Daniel Tarrazó-Serrano ${ }^{1}\left(\mathbb{0}\right.$, Sergio Castiñeira-Ibáñez ${ }^{1}{ }^{\circledR}$, Oleg V. Minin ${ }^{2}$, Pilar Candelas ${ }^{1}$, \\ Constanza Rubio ${ }^{1, *(D)}$ and Igor V. Minin ${ }^{3, *}$ \\ 1 Centro de Tecnologías Físicas, Universitat Politècnica de València, Camí de Vera s/n, 46022 València, Spain; \\ dtarrazo@fis.upv.es (D.T.-S.), sercasib@upvnet.upv.es (S.C.-I.), pcandelas@fis.upv.es (P.C.), \\ crubiom@fis.upv.es (C.R.) \\ 2 Tomsk State University, 30 Lenin Avenue, Tomsk, 634050, Russia; oleg.minin@ngs.ru \\ 3 Tomsk Politechnical University, 36 Lenin Avenue, Tomsk, 634050, Russia; prof.minin@gmail.com \\ * Correspondence: crubiom@fis.upv.es; prof.minin@gmail.com
}

Academic Editor: name

Version September 11, 2018 submitted to Preprints

\begin{abstract}
The image performance of acoustic and ultrasound sensors depends on several fundamental parameters such as depth of focus or spatial resolution. There are currently two different type of acoustic diffractive lenses: those which form a diffraction-limited spot with a shallow depth of focus (zone plates) and lenses which form an extended focus (quasi-Bessel beams). In this paper, we investigate a pupil-masked Soret zone plate which allows the tunability of a normalized angular spectrum. It is shown that the depth of focus and the spatial resolution can be modified, without changing the lens structure, by choosing the size of the amplitude pupil mask. This effect is based on the transformation of spherically converging waves into quasi-conical waves, due to the apodization of the central part of the zone plate. The theoretical analysis is verified with both numerical simulations and experimental measurements. A Soret zone plate immersed in water with $\mathrm{D} / 2 \mathrm{~F}=2.5$ and $\mathrm{F}=4.5 \lambda$, changes its depth of focus from $2.84 \lambda$ to $5.9 \lambda$ and the spatial resolution increases from $0.81 \lambda$ to $0.64 \lambda$ at a frequency of $250 \mathrm{kHz}$, by modifying the pupil mask dimensions of the Soret zone plate.
\end{abstract}

Keywords: ultrasonic lens; apodization; Soret zone plate

\section{Introduction}

The necessity of manipulating waves has been one of the main objectives of the scientific community. Lenses are devices that achieve, through different physical phenomena such as refraction or diffraction, beam focusing and modulating effects. In the acoustic field, lenses have been developed for different areas such as biomedicine, engineering, industry and those applications which require a reduced cost of acoustic lenses [1-11].

Due to the interest that these lenses arose, their design and improvement is currently a research subject. One type of acoustic lenses are those which change the refractive index among the medium known as Gradient-Index (GRIN) lenses using labyrinthine structures [12-14]. Phononic crystals [15], acoustic metamaterials [16] and acoustic resonators [17] have also been used in the implementation of acoustic lenses.

Fresnel Zone Plates (FZP), as a simple example of acoustic lens, have been used in this study due to their dimension and fabrication advantages [18-20]. FZPs are constructed alternating opaque and transparent acoustic rings and use the diffraction phenomenon to modulate and focus acoustic waves. These classic FZPs are also called Soret Zone Plates (SZPs) [21].

Calvo et al. developed and characterized a SZP for underwater ultrasounds, alternating opaque and transparent zones made of soft silicone rubber [22]. Results shown a main lobe widening but also, a small reduction of the first Side Lobe Level (SLL). A zone plate based on fractal structures was studied in [23]. 
Acoustic field diffraction produces a maximum pressure field located in the central axis, nonetheless the secondary lobes produce miss-definition on the focal point [24-26]. SLL and Depth of Focus (DoF) are one of the problems that SZP lenses have [27]. Although energy is concentrated at the Focal Length $\left(F_{L}\right)$, SLL levels in SZP lenses are not sufficient if the target application requires high precision.

This work is based on the study carried out by Minin et al. in the field of electromagnetism [28]. In that study, a modification on the distribution of energy modification at the focus was proposed. The authors showed that by choosing a radius or phase reference in the first SLL radii, the SLL ratio was improved $[29,30]$. These results can be transferred to the ultrasonic field. Later the concept of the reference radius was independently used in acoustics in [31], but without analyzing the image quality.

The quality of the image is determined by the beam distribution (lateral resolution, $R_{\text {lat }}$ ) and axial resolution, $R_{a x}$. For an arbitrary circular aperture at the focus, simplified expressions for lateral resolution, $R_{\text {lat }}$, and (DoF), are [32]:

$$
\begin{gathered}
R_{\text {lat }}=\alpha \lambda \frac{F}{D}=\alpha \lambda f^{\#} \\
D o F=\beta \lambda\left(\frac{F}{D}\right)^{2}=\beta \lambda f^{\# 2}
\end{gathered}
$$

Here, the ratio of the focal length to the diameter is the f-number $f^{\#}$. From Equations (1) and (2) it is followed that [33]:

$$
D o F=\frac{\beta}{\alpha^{2} \lambda} R_{l a t}^{2}
$$

Equation (1) is valid also in the focal area for a focused lens with a high numerical aperture [34]. One could see that depending on the choice of frequency and $f^{\#}$, the resolution can vary over several orders of magnitude.

To increase the depth of focus and spatial resolution many papers investigated acoustic Bessel beams due to the central core spot size of the Bessel beam, which is defined from the properties of the zeros of a Bessel function and is given by:

$$
R_{\text {lat }}=2.4048 / k_{r}
$$

where $k_{r}$ is a radial wave vector [35].

For example, a flat acoustic lens with an aperiodical structure to transform a divergent beam into a Bessel like beam was reported in [36]. Bessel beams of sound waves were also reported in [37,38]. However, they are not as broadly applied as in optics, which is perhaps related to the lack of convenient techniques of formation of such kind of acoustic waves. Acoustic Bessel beams have been excited using acoustical axicons [39], in analogy to the optical case. In any case, the most convenient way to form acoustic Bessel beams is by using annular transducer arrays [40,41]. The acoustic Bessel-like beam by means of an axisymmetric grating of rigid tori was reported in [42]. It could be mentioned that if Babinet's principle is considered, both approaches (FZP and rigid tori scatterers) are considered equivalent.

The present work proposes and demonstrates a new technique for acoustic quasi-Bessel beam formation using a planar structure based on SZPs [21]. Two concepts for a diffraction lens design are considered: based on the reference phase method and the amplitude pupil mask. It can be affirmed that, under specific conditions, part of a diffracted wave collimates producing an elongated focus. Numerical calculations using Finite Element Method (FEM) of acoustic waves propagating through such lenses were used to observe the complete acoustic field. Finally, the experimental verification of quasi-Bessel beam formation by two types of Soret Zone Plates is reported. 


\section{Mathematical Method and Simulations}

SZPs are based on ring sections known as Fresnel Regions. The purpose is to block the destructive contributions to the focus. Therefore, consecutive Fresnel Regions have a $\pi$ phase shift between zones. SZP's building parameters are $F_{L}$, the Number of Fresnel Zones $(N)$ and the working frequency $(f)$. The radii are obtained from the following Fresnel construction equation,

$$
r_{n}=\sqrt{n \lambda F_{L}+\left(\frac{n \lambda}{2}\right)}
$$

The reference radius is defined arbitrarily, on account that the properties of the zone plate are not modified from the point of view of geometrical optics [28]. Nevertheless, this reference radius is equal to modify the reference phase in the wave approximation [30]. This phase shift introduces the quasi-Fresnel radii concept. These radii are defined, for the plane incident wavefront, by the following equation,

$$
r_{n}=\sqrt{n \lambda \sqrt{F_{L}^{2}+r_{0}^{2}}+\left(\frac{n \lambda}{2}\right)^{2}+r_{0}^{2}}
$$

The study of the physical phenomena involved in the interaction between the lenses and the wave front requires a mathematical model that considers the boundary conditions of the problem. In the present work, the FEM method has been used through the commercial software COMSOL Multiphysics [43] to calculate the acoustic pressure distribution. This method generates a numerical solution by discretizing the model, solving Helmholtz's Partial Differential Equation,

$$
\nabla \cdot\left(-\frac{1}{\rho_{0}}(\nabla p)\right)=\frac{\omega^{2} p}{\rho_{0} c^{2}}
$$

One of the problems with the use of FEM, is the extensive usage of memory resources. To avoid them, axisymmetric models have been considered. This design, simplifies the calculation when considering that half-lens generates the rest of the model by turning $2 \pi$. Therefore, the degrees of freedom needed to obtain the numerical solution are reduced, decreasing the computation time. Underwater transmission is considered, and to solve Equation (7) typical water values of sound speed propagation $(c=1500 \mathrm{~m} / \mathrm{s})$ and density $\left(\rho=1000 \mathrm{~kg} / \mathrm{m}^{3}\right)$ are taken. Design frequency $(f)$ is $250 \mathrm{kHz}$, therefore wavelength $(\lambda)$ is $6 \mathrm{~mm}$. Lenses were defined as full rigid considering Neumann condition that specifies sound velocity in the boundary is zero. The exterior contours of the model emulate the Sommerfeld condition. This boundary condition turns into an infinitely host defining these contours as radiation boundaries. Mesh geometry was fixed in triangles with minimum element size of $\lambda / 14$ and maximum element size of $\lambda / 8$ to prevent numerical dispersion.

In the current paper, the lenses with high numerical aperture $(N A)$ were selected $(D / 2 F=2.5)$. Focusing of this device $(F=4.5 \lambda$ ) leads to a very compact beam with a short working distance. Below it has been considered and compared three types of SZPs based on acoustic lenses: classical SZP, zone plate with amplitude pupil mask (PSZP) and zone plate based on reference phase concept (RSZP). The simulation results are presented in self-normalized intensity maps with normalized axis to the wavelength as shown in Figure 1. Also, normalized intensity radial and longitudinal cuts along the $\mathrm{R}$ and the $\mathrm{Z}$ axis are shown in Figure 2, respectively.

From Figure 1 it is clear that the structures of focused acoustic fields in the focal area are different and in the cases of PSZP and RSZP have an extended focus. Moreover, from Equation (5) and Equation (6) it is followed that if $r_{0}$ in Equation (6) equal to $r_{n}$ from Equation (5), the Fresnel radii for classical Equation (5) and Reference phase SZP Equation (6) are equal above $r_{0}$. Thus, such PSZP and RSZP will give equal results for the focal area formation. The focused field in the cases of PSZP and RSZP has a characteristic structure for quasi-Bessel beams with intensity profiles that closely resemble the ideal $J_{0}^{2}$ transverse-intensity distribution of Bessel beams (Figure 1(d)). 

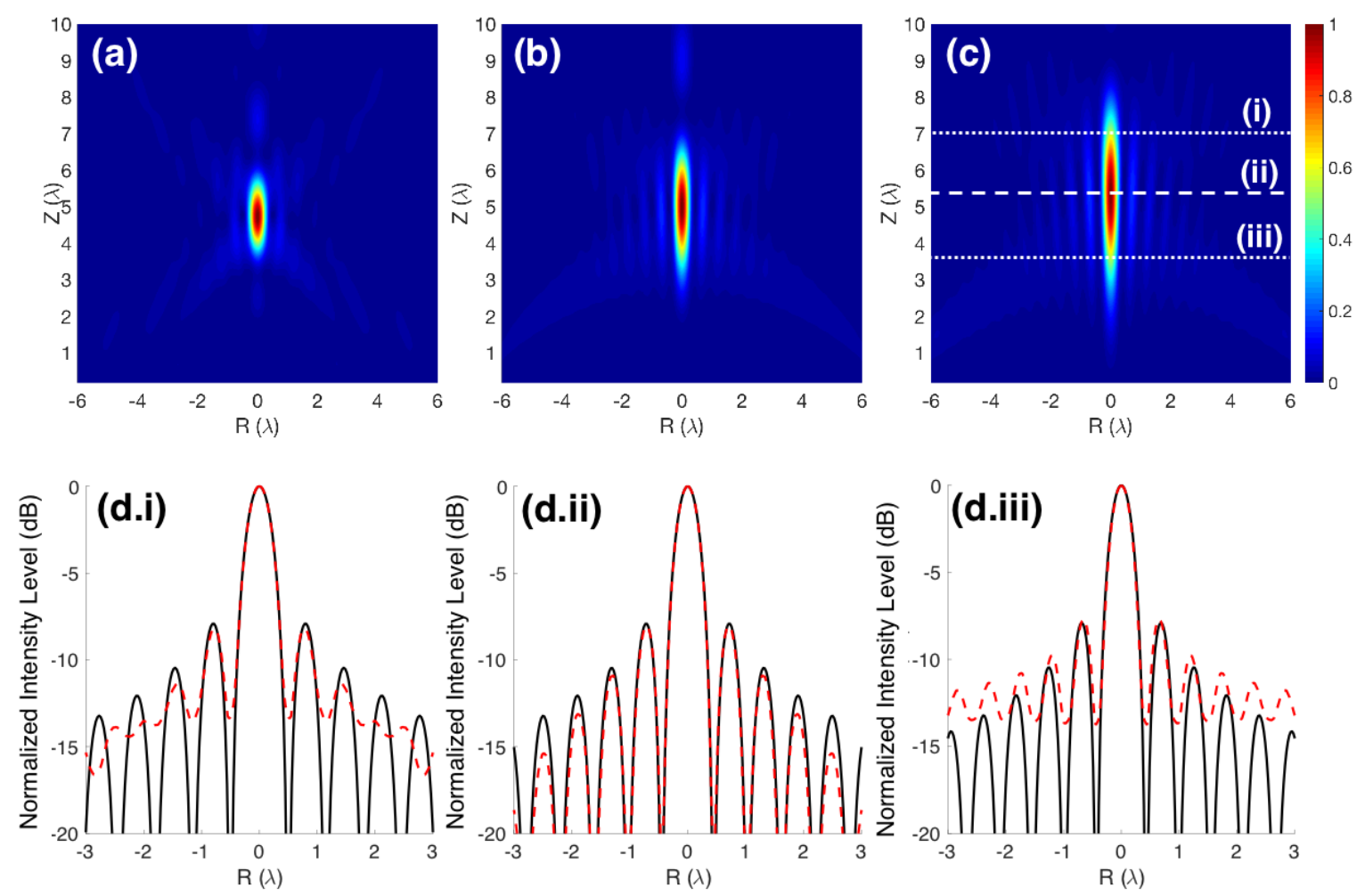

Figure 1. Self-normalized intensity maps to the maximum with $\mathrm{R}$ and $\mathrm{Z}$ axis normalized to $\lambda$ for (a) Soret Zone Plate (SZP), (b) Reference radius Soret Zone Plate (RSZP) and (c) Pupil Mask Soret Zone Plate (PSZP). (d) Black solid line is the Bessel $J_{0}^{2}$ function and dashed red line is the axial cut for PSZP lens at different positions: (d.i) corresponds to (i) line in Figure 1c, (d.ii) to (ii) line and (d.iii) to (iii) line respectively. The normalized Intensity Level (IL) in $\mathrm{dB}$ is represented abscise axis for all the cases. 

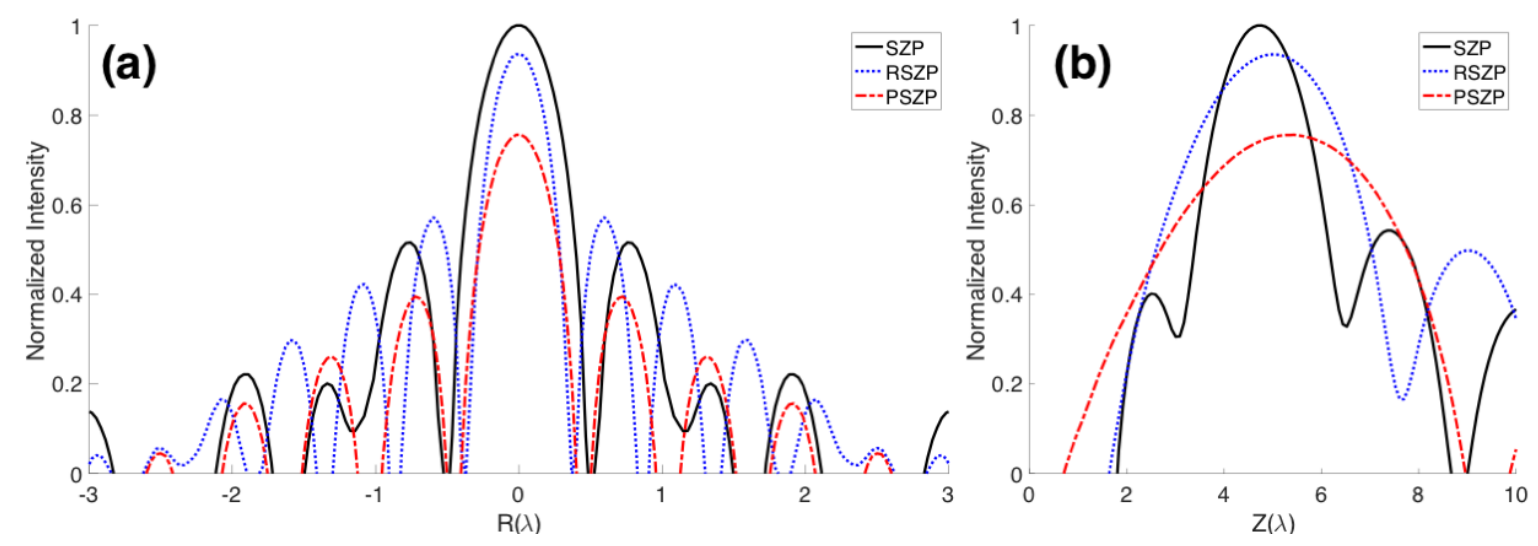

Figure 2. Numerical normalized intensity results for (a) $\mathrm{R}$ axis cut and (b) $\mathrm{Z}$ axis cut. Black solid line corresponds to classical Soret Zone Plate (SZP), blue dotted line corresponds to Reference Radius Soret Zone Plate (RSZP) and dash-dotted red line corresponds to the amplitude pupil masked Soret Zone Plate (PSZP).

Table 1. FLHM and FWHM comparison between numerical and experimental results.

\begin{tabular}{ccc}
\hline & FLHM $(\lambda)$ & FWHM $(\lambda)$ \\
\hline SZP & 2.84 & 0.81 \\
RSZP & 3.67 & 0.60 \\
PSZP & 5.94 & 0.64 \\
Bessel J & - & 0.64 \\
2 & 3.51 & 0.79 \\
FZP13Z7 (Experimental SZP) & 5.72 & 0.67 \\
\hline
\end{tabular}

The normalized intensity distributions along optical axis are presented in Figure 2. It can be observed that a maximum normalized intensity value is obtained for the SZP case. Normalized intensity values for RSZP and PSZP are 0.94 and 0.76 , respectively.

As the central zones for PSZP are covered, the central peak sharpens further. However, the relative side lobe intensity is increased. Thus, the central zones play an important role in reducing the background, while the outer zones cause the central peak to be sharpen. The diameter of the central maximum at the focal spot is less than the equivalent Airy disk, partly because the open areas are smaller than the corresponding Fresnel zones (see Table 1). In this Table it is compared the Full Length Half Maximum (FLHM) and Full Width Half Maximum (FWHM) of each lens. FLHM is defined as the distance along the longitudinal axis where the energy is reduced to the half. FWHM is equivalent to the FLHM but considering the transversal axis.

Compared to a classical SZP, an acoustic PSZP has the following main characteristics. Focus depth is larger than classical SZP; its resolution is better for point objects since the central maximum is narrower and it has a little more energy in the outer rings of the diffraction pattern (compared to the central maximum).

As it was mentioned above that PSZP and RSZP generate quasi-Bessel beams as shown in Figure 1. As for RSZP, in general, it can be mentioned that effectively, by increasing the diameter of the amplitude pupil, the diameter of the central spot is reduced, and the depth of focus is increased with the relative amplitude of the secondary lobes is increased. It is worth mentioning that in [32] a paraxial study of this phenomenon in optics was made in contrast to our study made for acoustic lens with high $N A$.

The effects of extended depth of focus with quasi-Bessel structure both for PSZP and RSZP may be described (to simplify the problem) as presented in Figure 3. 
(a)

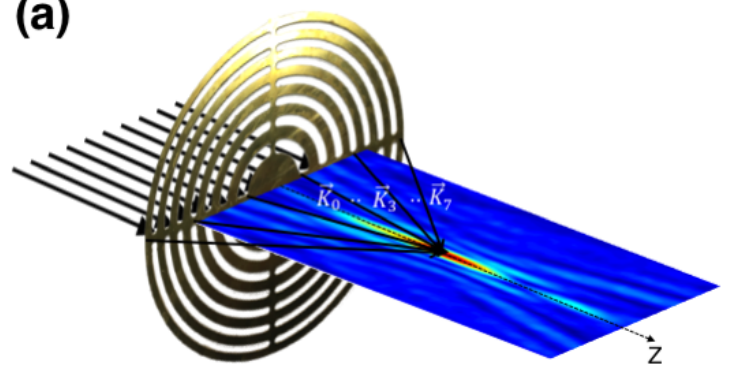

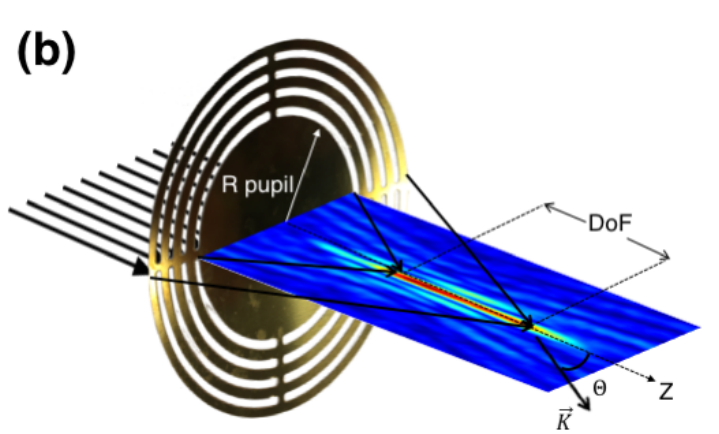

Figure 3. Beam formation scheme of (a) SZP and (b) PSZP.

The binary SZP lens consists of concentric dielectric ring, which can be treated as a quasi-periodic grating with different local grating constants at different radii. For single-point focusing, the normal incident wave is diffracted towards the designed focal point by these local gratings. The diffraction angles has a large range of different radii to ensure the formation of a focal spot (Figure 3a). The normalized radial spatial frequency $k_{r} / k$ is related to the angle $\theta$ as $k_{r} / k=\sin \theta$ where $k_{r}$ is the radial components of wave vector $k$, and $\theta$ is the angle between the wave vector and optical axis. For an ideal Bessel beam, the values of $k_{r}$, and $\theta$ are the same for all plane waves consisting of the beam. The range of the diffraction angle $\left(\theta_{i}\right)$ corresponds to the normalized angular spectrum bandwidth.

SZP with pupil masked central part and reference phase FZP with first reference radius equal to pupil mask dimension may be also considered as circular gratings. Due to the widths of zones are almost constant so PSZP and RSZP lenses are similar to ring diffraction grating. Thus the local gratings diffract the incident waves towards different points on the optical axis with a small range of diffraction angle (Figure $3 b$ ).

The interference pattern is a 2D Bessel function of the first kind, located at the focal distance. Therefore, the local grating constant $d(r)$ at radius $r$ can be obtained by the grating equation, $d(r) \sin \theta(r)=\lambda$, where $\lambda$ is the incident wavelength. Thus, the angular spectrum of the acoustic lens based on the SZP is controlled by the selection of the pupil mask size. It could be noted that due to acoustical lens is immersed in water the angular spectrum is compressed according to refractive index of a medium.

Most of the focused wave is diffracted from the outer rings and the first-order diffraction beams intercept the optical axis and form the acoustical needle, which is similar to the formation of a quasi-non-diffracting beam with conical lenses.

It could be noted that in the optical band, the pupil mask method was used to block the light through the central part in the point-focusing super-oscillatory lenses to achieve a DoF as long as $5 \lambda-20 \lambda$. However, the realization of such an extended DoF was at the expense of degradation in the focus transverse size [44,45].

\section{Experimental Results}

In order to carry out the experimental measurements, the SZP and the PSZP have been implemented in brass using a mechanization process. The material selected for its construction is brass because it has a relatively low transmission coefficient and behaves as an opaque sound material as shown in the Figure 4.

All the measures have been done using an automated full precision measurement system in order to validate and compare the proposed lenses. This well-know system consists in a fixed ultrasonic transducer and a 3D positioned hydrophone [46]. This systems grants precise and reliable results with $1 \times 1 \mathrm{~mm}^{2}$ scanning. The transducer used is a $250 \mathrm{kHz}$ Imasonic piston with $32 \mathrm{~mm}$ of active diameter and the needle hydrophone is a MPM1/1 from Precision Acoustic Ltd. made of polyvinylidene fluoride 
171 172

with a diameter of $1.5 \mathrm{~mm}$. Flat transfer function between 0.2 and $15 \mathrm{MHz}$ provides accuracy on the measurements.

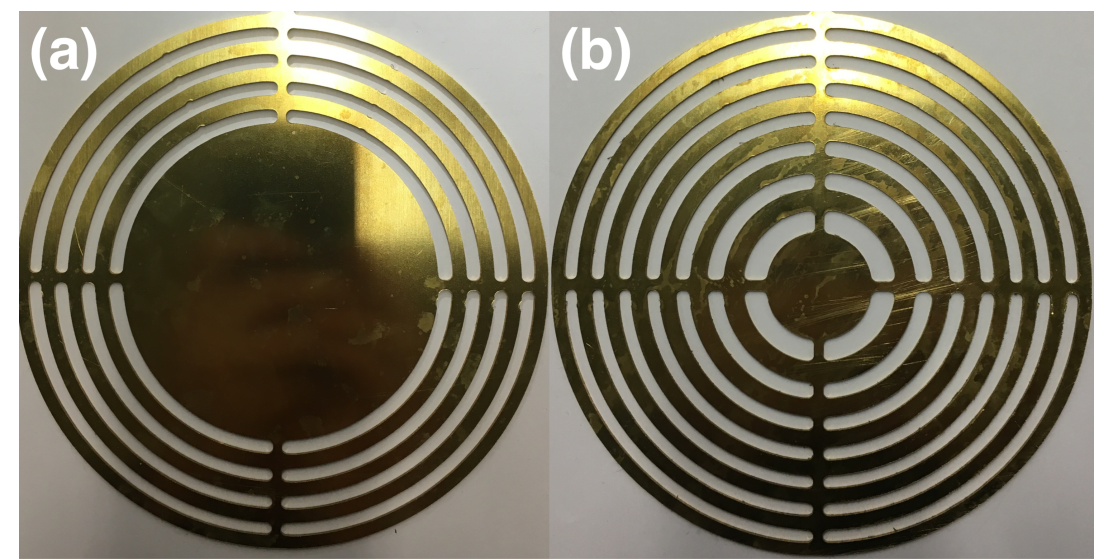

Figure 4. Implemented lenses in brass for experimental results. Where (a) corresponds to the experimental classical Soret Zone Plate (FZP13Z7) and (b) corresponds to the implemented amplitude Pupil Masked Soret Zone Plate (PM13Z4).

Figure 5 shows the experimental self-normalized intensity maps for Figure 5 a implemented SZP (FZP13Z7) and Figure 5b implemented PSZP (PM13Z4). The axial and longitudinal normalized intensity cuts are shown in Figure 6. Figure 6a corresponds to axial or R-axis cut and can be seen that the consideration of a pupil generates a distribution of energy along the longitudinal axis narrowing the beam and generating a quasi-Bessel beam distribution. Experimental results are in good agreement with the presented numerical results.
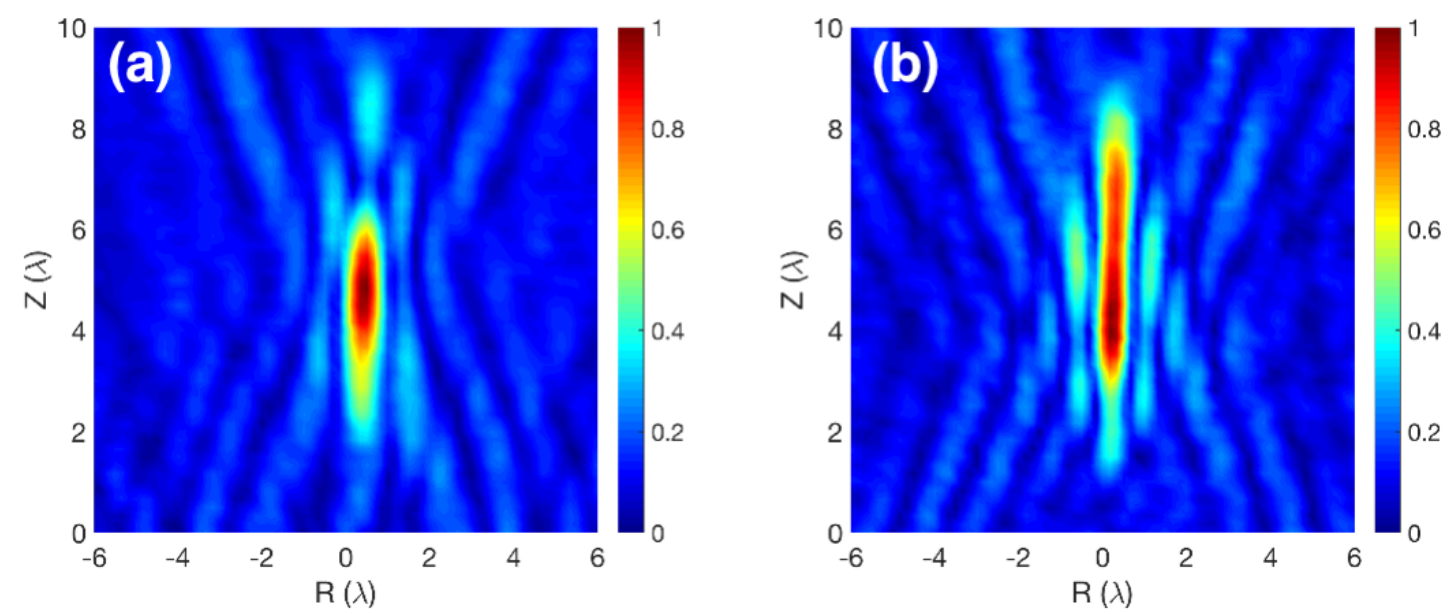

Figure 5. Experimental self-normalized intensity maps results for implemented lenses. Where (a) corresponds to the experimental classical Soret Zone Plate (FZP13Z7) and (b) corresponds to the implemented amplitude Pupil Masked Soret Zone Plate (PM13Z4). 

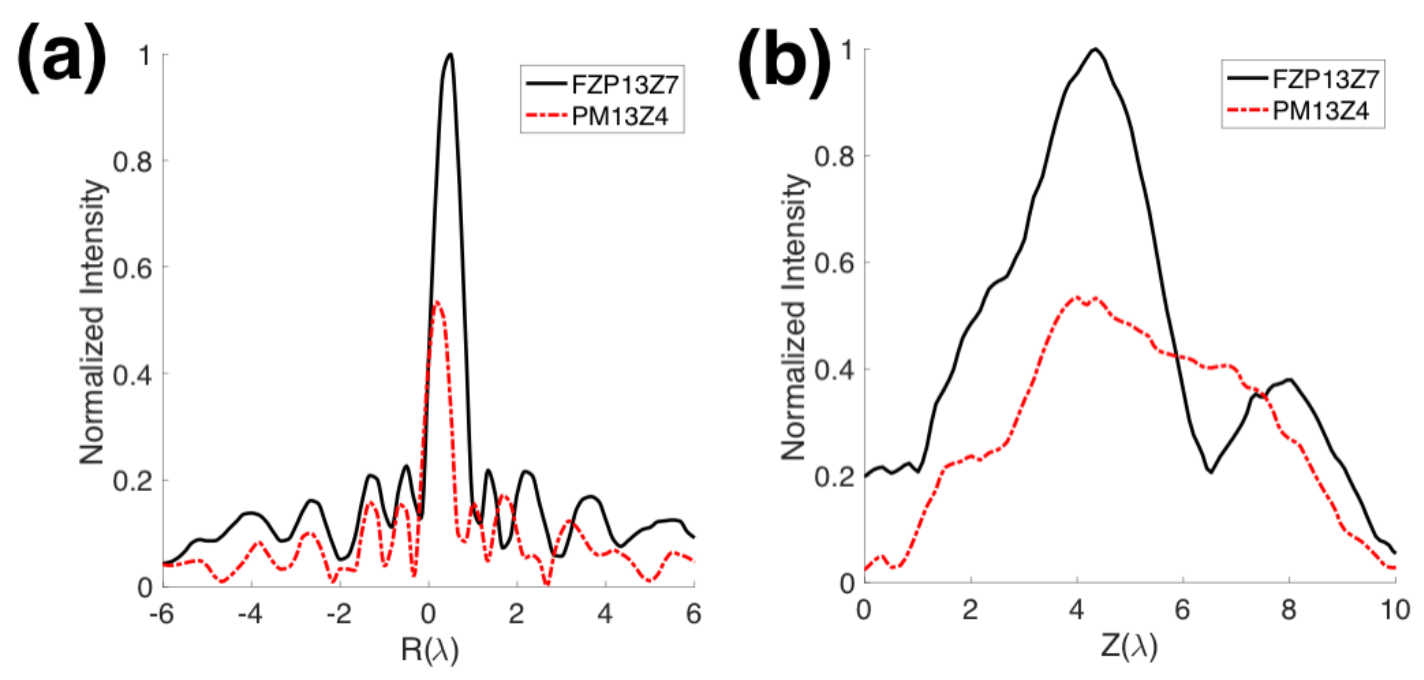

Figure 6. Experimental normalized intensity results for both implemented lenses. Axial and longitudinal axis are shown, where (a) corresponds to R-axis cuts and (b) corresponds to Z-axis cuts.

\section{Conclusion}

In this work, we have proposed an improvement over a classical SZP introducing an amplitude pupil mask. The idea of a normalized angular tunable acoustic spectrum has been verified. The design of a quasi-non-diffracting beam with sub-wavelength transverse size is achieved using a pupil-masked or reference phase SZP. This design has been verified using numerical models and the experimental measurements in controlled conditions. When an amplitude pupil masked is used, a quasi-Bessel beam instead of a diffraction spot focus is obtained. This effect is produced by modifying the spherically converging waves into quasi-conical waves. Therefore, a quasi-Bessel beam distribution is obtained when an amplitude pupil masked SZP is used.

With the amplitude pupil masked SZP proposed in this paper, a spatial resolution enhancement from $0.81 \lambda$ to $0.64 \lambda$ is accomplished. In addition, depth of focus is increased from $2.84 \lambda$ to $5.94 \lambda$ compared with the classical SZP. The experimental results are in good agreement with the numerical ones. The obtained results allows to modulate the acoustic beam without changing the lenses. Therefore, the same SZP could be used for different targets by adding an amplitude pupil mask. In consequence, these results confer great versatility to SZP lenses. This type of PSZP could have applications in different areas where compromise between $D o F$ and partial resolution is needed. As happens in 3D imaging, ultrasonic sensors with long depth of focus eliminates the need for depth scanning making this technique considerably faster.

Author Contributions: Igor V. Minin and Constanza Rubio coordinated the theoretical development, participating in the establishment of the theory principles used in this work, as well as in the drafting of the manuscript. Constanza Rubio and Daniel Tarrazó-Serrano coordinated experimental development. Oleg V. Minin and Sergio Castiñeira-Ibáñez developed part of the theory used and designed some characterization. Pilar Candelas participated in the analysis of the state of art, as well as the project administration and funding acquisition.

Acknowledgments: This work has been supported by Spanish MINECO (TEC2015-70939-R) and was partially supported by Tomsk Polytechnic University Competitiveness Enhancement Program.

Conflicts of Interest: The authors declare no conflict of interest. 
1. Pignoli, P.; Tremoli, E.; Poli, A.; Oreste, P.; Paoletti, R. Intimal plus medial thickness of the arterial wall: A direct measurement with ultrasound imaging. Circulation 1986, 74, 1399-1406. doi:10.1161/01.CIR.74.6.1399.

2. Amemiya, I.; Yagi, H.; Mori, K.; Yamamoto, N.; Saitoh, S.; Tanuma, C.; Hirahara, S. Ink Jet Printing with Focused Ultrasonic Beams. Recent Progress in Ink Jet Technologies II; Vol. 5, Society for Imaging Science and Technology, 1999; pp. 274-279.

3. McCann, D.; Forde, M. Review of NDT methods in the assessment of concrete and masonry structures. NDT and E International 2001, 34.

4. Li, J.T.; Han, J.F.; Yang, J.H.; Li, T.S. An efficient synthesis of 3, 4-dihydropyrimidin-2-ones catalyzed by $\mathrm{NH} 2 \mathrm{SO} 3 \mathrm{H}$ under ultrasound irradiation. Ultrasonics Sonochemistry 2003, 10, 119-122.

5. Albu, S.; Joyce, E.; Paniwnyk, L.; Lorimer, J.; Mason, T. Potential for the use of ultrasound in the extraction of antioxidants from Rosmarinus officinalis for the food and pharmaceutical industry. Ultrasonics Sonochemistry 2004, 11, 261-265.

6. Illing, R.; Kennedy, J.; Wu, F.; Ter Haar, G.; Protheroe, A.; Friend, P.; Gleeson, F.; Cranston, D.; Phillips, R.; Middleton, M. The safety and feasibility of extracorporeal high-intensity focused ultrasound (HIFU) for the treatment of liver and kidney tumours in a Western population. British journal of cancer 2005, 93, 890.

7. Vilkhu, K.; Mawson, R.; Simons, L.; Bates, D. Applications and opportunities for ultrasound assisted extraction in the food industry-A review. Innovative Food Science \& Emerging Technologies 2008, 9, 161-169.

8. Schonbrun, E.; Rinzler, C.; Crozier, K.B. Microfabricated water immersion zone plate optical tweezer. Applied Physics Letters 2008, 92, 071112.

9. Hon, S.; Kwok, K.; Li, H.; Ng, H. Self-focused acoustic ejectors for viscous liquids. Review of scientific instruments 2010, 81, 065102.

10. Calvo, D.C.; Thangawng, A.L.; Nicholas, M.; Layman, C.N. Thin Fresnel zone plate lenses for underwater acoustics: Modeling and experiments. OCEANS'15 MTS/IEEE Washington 2015.

11. Tu, Y.L.; Chen, S.J.; Hwang, Y.R. Design of Fresnel Lens-Type Multi-Trapping Acoustic Tweezers. Sensors 2016, 16, 1973.

12. Welter, J.T.; Sathish, S.; Christensen, D.E.; Brodrick, P.G.; Heebl, J.D.; Cherry, M.R. Focusing of longitudinal ultrasonic waves in air with an aperiodic flat lens. The Journal of the Acoustical Society of America 2011, 130, 2789-2796. doi:10.1121/1.3640841.

13. Li, Y.; Liang, B.; Tao, X.; Zhu, X.F.; Zou, X.Y.; Cheng, J.C. Acoustic focusing by coiling up space. Applied Physics Letters 2012, 101.

14. Peng, P.; Xiao, B.; Wu, Y. Flat acoustic lens by acoustic grating with curled slits. Physics Letters, Section A: General, Atomic and Solid State Physics 2014, 378, 3389-3392. doi:10.1016/j.physleta.2014.09.042.

15. Cervera, F.; Sanchis, L.; Sánchez-Pérez, J.V.; Martínez-Sala, R.; Rubio, C.; Meseguer, F.; López, C.; Caballero, D.; Sánchez-Dehesa, J. Refractive Acoustic Devices for Airborne Sound. Physical Review Letters 2002, $88,023902$.

16. Guenneau, S.; Movchan, A.; Pétursson, G.; Ramakrishna, S.A. Acoustic metamaterials for sound focusing and confinement. New Journal of Physics 2007, 9.

17. Moleron, M.; Serra-Garcia, M.; Daraio, C. Acoustic Fresnel lenses with extraordinary transmission. Applied Physics Letters 2014, 105.

18. Farnow, S.; Auld, B. Acoustic Fresnel zone plate transducers. Applied Physics Letters 1974, 25, 681-682.

19. O'Shea, D.C.; Suleski, T.J.; Kathman, A.D.; Prather, D.W. Diffractive optics: design, fabrication, and test; Vol. 62, Spie Press Bellingham, WA, 2004.

20. Zhang, S.; Yin, L.; Fang, N. Focusing ultrasound with an acoustic metamaterial network. Physical Review Letters 2009, 102.

21. Soret, J. Ueber die durch Kreisgitter erzeugten Diffractionsphänomene. Annalen der Physik 1875, 232, 99-113.

22. Calvo, D.C.; Thangawng, A.L.; Nicholas, M.; Layman, C.N. Thin Fresnel zone plate lenses for focusing underwater sound. Applied Physics Letters 2015, 107.

23. Castiñeira-Ibáñez, S.; Tarrazó-Serrano, D.; Rubio, C.; Candelas, P.; Uris, A. An Ultrasonic Lens Design Based on Prefractal Structures. Symmetry 2016, 8, 28. 
24. Bashford, A.G.; Schindel, D.W.; Hutchins, D.A.; Wright, W.M.D. Field characterization of an air-coupled micromachined ultrasonic capacitance transducer. Journal of the Acoustical Society of America 1997, 101, 315-322.

25. Kanevsky, I.N. Focusing of Sound and Ultrasound Waves [in Rusian]; Nauka, Moscow, 1977.

26. Hiroshi, S.; Noriyoshi, C.; Jun Ichi, K. Acoustical Imaging v.17; Plenum Press, New York and London, 1989.

27. Minin, I.V.; Minin, O.V. Basic principles of fresnel antenna arrays; Vol. 19, Springer Science \& Business Media, 2008.

28. Minin, I.V.; Minin, O.V. Control of focusing properties of diffraction elements. Soviet Journal of Quantum Electronics 1990, 20, 198.

29. Webb, G.; Minin, I.; Minin, O.V. New technique to suppress sidelobe clutter in perimeter security systems. International Journal of High Speed Electronics and Systems 2007, 17, 367-382.

30. Minin, I.V.; Minin, O.V. Reference phase in diffractive lens antennas: A review. Journal of Infrared, Millimeter, and Terahertz Waves 2011, 32, 801.

31. Clement, G.T.; Nomura, H.; Kamakura, T. Ultrasound Field Measurement Using a Binary Lens. IEEE Transactions on Ultrasonics, Ferroelectrics and Frequency Control 2015, 62, 350-359. doi:TUFFC.2014.006800.

32. Born, M.; Wolf, E. Principles of optics: electromagnetic theory of propagation, interference and diffraction of light; Elsevier, 2013.

33. Minin, O.V.; Minin, I.V. Diffractive optics of millimeter waves; IOP Publisher, London, 2004.

34. Minin, I.V.; Minin, O.V.; Gagnon, N.; Petosa, A. Investigation of the resolution of phase correcting Fresnel lenses with small focal length-to-diameter ratio and subwavelength focus. Proceeding of the EMTS 2007, pp. $26-28$.

35. McGloin, D.; Dholakia, K. Bessel beams: diffraction in a new light. Contemporary physics 2005, 46, 15-28.

36. Xu, Z.; Xu, W.; Qian, M.; Cheng, Q.; Liu, X. A flat acoustic lens to generate a Bessel-like beam. Ultrasonics 2017, 80, 66-71.

37. Hsu, D.; Margetan, F.; Thompson, D. Bessel beam ultrasonic transducer: Fabrication method and experimental results. Applied physics letters 1989, 55, 2066-2068.

38. Lu, J.Y.; Greenleaf, J.F. A study of two-dimensional array transducers for limited diffraction beams. IEEE transactions on ultrasonics, ferroelectrics, and frequency control 1994, 41, 724-739.

39. Katchadjian, P.; Desimone, C.; Garcia, A. Application of axicon lenses in ultrasonic techniques. AIP Conference Proceedings. AIP, 2010, Vol. 1211, pp. 1043-1050.

40. Masuyama, H.; Yokoyama, T.; Nagai, K.; Mizutani, K. Generation of Bessel beam from equiamplitude-driven annular transducer array consisting of a few elements. Japanese journal of applied physics 1999, 38, 3080 .

41. Masuyama, H.; Mizutani, K. Acoustic Beam Scanning Using Annular Transducer Array Introducing Decentering Operation. JSME International Journal Series C Mechanical Systems, Machine Elements and Manufacturing 2006, 49, 681-686.

42. Jimenez, N.; Romero-García, V.; Picó, R.; Cebrecos, A.; Sánchez-Morcillo, V.J.; Garcia-Raffi, L.; Sánchez-Pérez, J.V.; Staliunas, K. Acoustic Bessel-like beam formation by an axisymmetric grating. EPL (Europhysics Letters) 2014, 106, 24005.

43. COMSOL-Multiphysics. COMSOL-Multiphysics User Guide (version 4.3a). COMSOL User Guide (version 4.3a) 2012, pp. 39-40.

44. Qin, F.; Huang, K.; Wu, J.; Jiao, J.; Luo, X.; Qiu, C.; Hong, M. Shaping a subwavelength needle with ultra-long focal length by focusing azimuthally polarized light. Scientific reports 2015, 5, 9977.

45. Roy, T.; Rogers, E.T.; Yuan, G.; Zheludev, N.I. Point spread function of the optical needle super-oscillatory lens. Applied Physics Letters 2014, 104, 231109.

46. Rubio, C.; Fuster, J.M.; Castiñeira-Ibáñez, S.; Uris, A.; Belmar, F.; Candelas, P. Pinhole Zone Plate Lens for Ultrasound Focusing. Sensors 2017, 17, 1690. 